Macroscopic Subdivision of Silica Aerogel Collectors for Sample Return Missions

H. A. Ishii, J. P. Bradley

September 23, 2005

Meteoritics and Planetary Science 
This document was prepared as an account of work sponsored by an agency of the United States Government. Neither the United States Government nor the University of California nor any of their employees, makes any warranty, express or implied, or assumes any legal liability or responsibility for the accuracy, completeness, or usefulness of any information, apparatus, product, or process disclosed, or represents that its use would not infringe privately owned rights. Reference herein to any specific commercial product, process, or service by trade name, trademark, manufacturer, or otherwise, does not necessarily constitute or imply its endorsement, recommendation, or favoring by the United States Government or the University of California. The views and opinions of authors expressed herein do not necessarily state or reflect those of the United States Government or the University of California, and shall not be used for advertising or product endorsement purposes. 


\title{
MACROSCOPIC SUBDIVISION OF SILICA AEROGEL COLLECTORS FOR SAMPLE RETURN MISSIONS
}

\author{
Hope A. Ishii* and John P. Bradley \\ Institute of Geophysics and Planetary Physics, L-413, Lawrence Livermore National Laboratory, \\ 7000 East Avenue, Livermore, CA 94550 \\ *Corresponding author. E-mail: hope.ishii@1lnl.gov
}




\begin{abstract}
Silica aerogel collector tiles have been employed for the collection of particles in low Earth orbit and, more recently, for the capture of cometary particles by NASA's Stardust mission. Reliable, reproducible methods for cutting these and future collector tiles from sample return missions are necessary to maximize the science output from the extremely valuable embedded particles. We present a means of macroscopic subdivision of collector tiles by generating large-scale cuts over several centimeters in silica aerogel with almost no material loss. The cut surfaces are smooth and optically clear allowing visual location of particles for analysis and extraction. This capability is complementary to the smaller-scale cutting capabilities previously described [Westphal (2004), Ishii (2005a, 2005b)] for removing individual impacts and particulate debris in tiny aerogel extractions. Macroscopic cuts enable division and storage or distribution of portions of aerogel tiles for immediate analysis of samples by certain techniques in situ or further extraction of samples suited for other methods of analysis.
\end{abstract}




\section{INTRODUCTION}

In January of 2006, the NASA Stardust mission will bring to Earth the first solid samples ever retrieved from the coma of a comet [Brownlee (2003)]. These samples were collected during a fly-by of the comet Wild 2 in early 2004 and are anticipated to consist of micron-sized particles embedded in silica aerogel collector tiles. The impact speed of the particles was $6.2 \mathrm{~km} / \mathrm{s}$, a hypervelocity expected to allow a relatively high degree of preservation of the terminal particles. The actual condition of the captured particles in the aerogel will remain unknown until the mission returns, but essential to any scenario is a capability for extracting the sample from the collector. The Orbital Debris Collector Experiment exposed silica aerogel tiles on the Mir and US space stations to collect manmade and extraterrestrial particles in low earth orbit [Hörz (2000)]. This has provided one opportunity to explore how to extract samples from bulk aerogel tiles for analysis. Light gas gun hypervelocity shots of standard powders into aerogel are another source of test materials. For some early stages of the Stardust analysis, it is of interest to be able to preserve the impact track together with the terminal particulate material since valuable chemical information from less refractory materials may be deposited along the impact track. Considerable effort has been invested in developing methods for extracting tiny sections of aerogel, fractions of a millimeter on a side, containing individual particles and their associated impact tracks [Westphal (2004), Ishii (2005a, 2005b)]. However, many analysis techniques do not require such fine subdivision of the aerogel, and some offer major benefits for locating particles and prescreening by mapping larger sections of aerogel. Synchrotron x-ray techniques, for example, will allow mapping of major element distributions in centimeter-thick sections of aerogel followed by more in-depth fluorescence and spectroscopic studies of impact tracks and 
particles of interest. For these techniques, subdivided aerogel in larger portions with thicknesses on the order of several millimeters and lateral dimensions that might extend out to the full aerogel tile dimensions $(2-4 \mathrm{~cm})$ is more ideal. A means of macroscopic subdivision of the silica aerogel collector tiles is also desirable to facilitate preservation of portions of tiles and transport and distribution of other portions. Larger subdivided slices or blocks can be readily distributed for analysis or further divided, and individual tracks extracted for other analyses (for example, full chemical analysis of debris distributed along an impact track) or for isolation of terminal particles for TEM, nanoSIMS and other small particle analyses.

Several approaches for large-scale cutting of aerogel tiles have been tested by others. (See, for example, Tsou, 2005 LPSC). These approaches include diamond-impregnated wire saws, rotating saws, laser-cutting and ion milling. The saw- and laser-cutting approaches have the advantages of being fast and inexpensive. The major disadvantages to all of these approaches are high loss of surrounding aerogel material (kerf) and/or loss of optical clarity that obstructs further extraction and analysis of impacted material. For cutting hypervelocity impacts from aerogel, the reported methods achieve cutting depths of only a few millimeters over lateral distances of similar scale [Westphal (2004), Ishii (2005a, 2005b)].

We report here an extension, under development, of the ultrasonic microblade cutting method [Ishii (2005a, 2005b)] to produce macroscopic cuts in aerogel for the purpose of subdividing bulk aerogel tiles into manageable sub-sections for distribution, early survey analysis and eventual further subdivision and terminal particle extraction. These sub-sections may have thicknesses as thin as a millimeter over lateral distances of many centimeters. The cut surfaces 
have low surface roughness and high optical clarity allowing clear viewing of subsurface particles for further sample mining.

\section{METHOD AND RESULTS}

We have used an "ultrasonic macroblade," a steel utility-knife blade driven at ultrasonic frequencies, mounted on a micromanipulator for fine motion control to generate macroscopic cuts with smooth cut surfaces, exceptional optical clarity and almost no material loss. 2-cm long, steel blades were laser-cut en masse into a utility-knife shape from 100-micron thick, doubleedged, high carbon steel razor blades (Electron Microscopy Sciences), and cutting debris was gently filed off avoiding damage to the sharp cutting edge. Some blades showed warping at the tip due to thermal processing and were discarded. Figure 1 shows the blade used for this work. After mounting, it has an effective cutting length of $1.7 \mathrm{~cm}$, a width of $2 \mathrm{~mm}$ and a thickness of 100 microns with a $20^{\circ}$ angle of the cutting edge to the spine of the blade. The ultrasonic frequency is produced by the piezo-driver of the MicroDissector (Eppendorf) which fits on standard micromanipulators. Further details of the setup used for this work may be found in Ishii MAPS (2005).

Cuts are made by driving the macroblade at an ultrasonic frequency of approximately $32 \mathrm{kHz}$ and 100\% amplitude (full power). For each blade, the frequency is fine-tuned for clean cutting. Although the ultrasonic driver is nominally driving the blade longitudinally with an amplitude of 1.5 microns, this frequency results in near-resonance excitation of the blade that gives out-ofplane motion. For the blade shown in Figure 1, the out-of-plane motion is approximately 150 
microns in air and is considerably damped in the aerogel. (The diamond microblades [Ishii MAPS (2005)] used for extraction of individual impact tracks have out-of-plane motion on the order of 5-10 microns in air.) The result is localized breaking up and compression of the aerogel's silica network with little friction. Another resonance is present near $24 \mathrm{kHz}$, but the amplitude of out-of-plane motion is smaller, and sticking is more likely. Drawing the blade through the aerogel with no ultrasonic excitation results in tearing. The optimum smooth cut surface is obtained by drawing the ultrasonic macroblade across the surface of the aerogel. After each pass, the blade is lifted above the aerogel surface to return to the starting position, and the next cutting pass is made starting from a deeper position in the aerogel bulk. Aerogel tearing is minimized by adjusting the step size in depth and the cutting speed. For $10 \mathrm{mg} / \mathrm{cc}$ silica aerogel, steps of up to 300 microns can be used; for $20 \mathrm{mg} / \mathrm{cc}$ silica aerogel, the step size is reduced to 100 microns. Figure 2 illustrates a block of aerogel cut from the edge of a silica aerogel tile (10 $\mathrm{mg} / \mathrm{cc}$ density, 9 millimeter thickness). The entire block was extracted in about an hour and a half, and cutting time can be optimized for the specific aerogel density (or density profile) of the tiles being subdivided. Figure 3 shows an additional cut made through the top half of the aerogel block in order to embed a microthermocouple intended for synchrotron x-ray heating experiments. Cuts several centimeters in length can be readily made, and cutting depth appears to be limited only by the effective blade length so far. It is possible to make longer blades for cuts $2 \mathrm{~cm}$ deep, the depth of Stardust collector tiles, to cut off slices of aerogel. Alternatively, shallower cuts can be made from both sides of the tile to release a slice, a method that has been used successfully. 
It should be noted that ultrasonic frequencies can be applied to other tools besides blades. Silica needles, for example, may be used to generate tunnels in aerogel, an approach which may be useful for quickly extracting individual particles from subdivided tiles. The tunnel size is dependent on the needle shape in part due to its resulting stiffness.

\section{MATERIAL CHOICE}

Stainless steel is a durable choice for ultrasonic macroblades; however, there are concerns associated with this material. Since many extraterrestrial particles are magnetic, one concern with steel blades is their ferromagnetic properties. This issue can be addressed by demagnetizing (degaussing) the blades in an $\mathrm{AC}$ magnetic field [Brownlee, private communication].

Contamination is a more critical concern when dealing with micron- and submicron-sized particulate samples. Steel will wear with long-term use, and although stainless steel particles can be ruled out by chemical signature, the addition of particulates from the cutting tool onto the aerogel surface - or even into the aerogel surface for techniques generating rough surface cuts is highly undesirable. For macroscopic cuts to subdivide larger tiles, however, some degree of surface contamination may be acceptable when particles and any associated tracks selected for analysis are extracted from below the surface.

We have explored alternative blade materials such as CVD diamond and sapphire that would pose less contamination risk. Regrettably, neither material withstands ultrasonic excitation without breaking. Natural diamond is a proven material for this application (as in ultrasonic 
diamond microblades ref.) that does not shed particles, but at the length required for cutting $2 \mathrm{~cm}$ thick tiles (minimum $1 \mathrm{~cm}$ depth), it is a very expensive alternative.

\section{SUMMARY}

For sample return missions employing silica aerogel collector tiles, such as NASA's Stardust mission to collect cometary particles, methods for cutting these tiles from sample return missions are vital to enable detailed study of the embedded particles. These methods must permit clean and controlled cutting of the fragile silica aerogel. To meet this need, several approaches have been tested, and past methods have suffered from loss of optical clarity as well as material on either side of the cut. We have presented ultrasonic macroblades, an extension of the ultrasonic diamond microblade technique [Ishii (2005a, 2005b)], to generate large-scale cuts for subdividing silica aerogel collector tiles used in particle capture on sample return missions. Application of ultrasonic vibrations to the macroblades provides a relatively quick tool for producing smooth cut surfaces with exceptional optical clarity and almost no material loss over centimeter distances permitting cuts over the length of a Stardust collector tile. Each subdivided portion of a given collector tile can be stored or distributed for in situ analysis (by x-ray techniques, for example) or for extraction of individual impact tracks and isolation of intact particles for in-depth analysis. Details of the cutting mechanism and parameters and the choice of blade materials have been discussed. The technique is complementary to the small-scale cutting techniques already described [Westphal (2004), Ishii (2005a, 2005b)] for extracting individual impact tracks. 


\section{Acknowledgements}

This work was performed under the auspices of the U.S. Department of Energy, NNSA by UC, Lawrence Livermore National Laboratory under contract No. W-7405-Eng-48. This work was performed as part of the Bay Area Particle Analysis Consortium (BayPAC) and was supported by NASA Grant NNH04AB49I. We thank Peter Tsou for discussions on the need for large-scale cutting and on cutting mechanisms and for general encouragement, and we acknowledge Eric Ziebarth for assistance with laser-cutting of the macroblades.

\section{Editorial Handling -}

\section{REFERENCES}

Brownlee D.E., Tsou P., Anderson J.D., Hanner MS., Newburn R. L., Sekanina Z., Clark B.C., Hörz F., Zolensky M.E., Kissel J., McDonnell J.A.M., Sandford S.A. and Tuzzolino A.J. 2003. Stardust: Comet and interstellar dust sample return mission. Journal of Geophysical Research 108: SRD 1-1-1-15.

Hörz F., Zolensky M. E., Bernhard R. P., See T. H. and Warren J. L. 2000. Impact features and projectile residues in aerogel exposed on Mir. Icarus 147: 559-579.

Ishii, H. A., Graham G. A., Kearsley A. T., Grant P. G., Snead C. J. and Bradley J. P. 2005. Ultrasonic micro-blades for the rapid extraction of impact tracks from aerogel. $36^{\text {th }}$ Lunar and Planetary Science Conference, CD-ROM: abstract 1387. 
Ishii, H. A., Graham G. A., Kearsley A. T., Grant P. G., Snead C. J. and Bradley J. P. 2005. Rapid extraction of dust impact tracks from silica aerogel by ultrasonic micro-blades. To be published, Meteoritics \& Planetary Science.

Tsou, P., Brownlee D. E., Glesias R., Grigoropoulos C. P. and Weschler M. 2005. Cutting silica aerogel for particle extraction. $36^{\text {th }}$ Lunar and Planetary Science Conference, CD-ROM: abstract 2307.

Westphal A. J., Snead C., Butterworth A., Graham G. A., Bradley J. P., Bajt S., Grant P. G., Bench G., Brennan S. and Pianetta P. 2004. Aerogel keystones: Extraction of complete hypervelocity impact events from aerogel collectors. Meteoritics \& Planetary Science 39: 1375-1386.

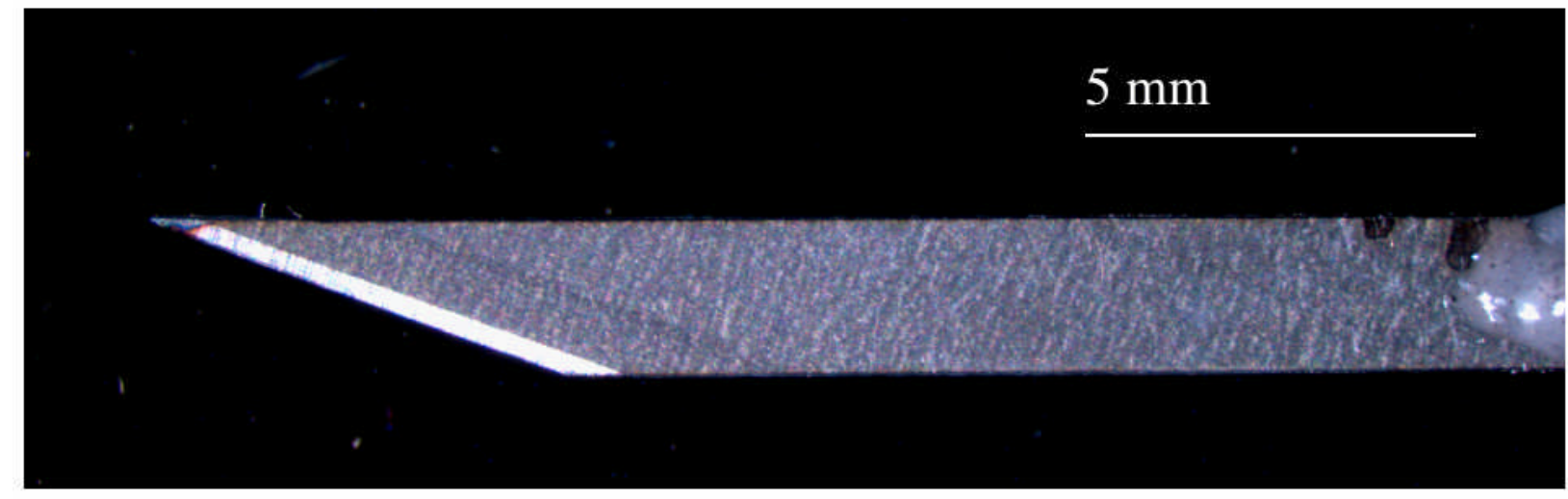

Figure 1. Macroblade laser-cut from a 100-micron thick breakable steel razor blade. 


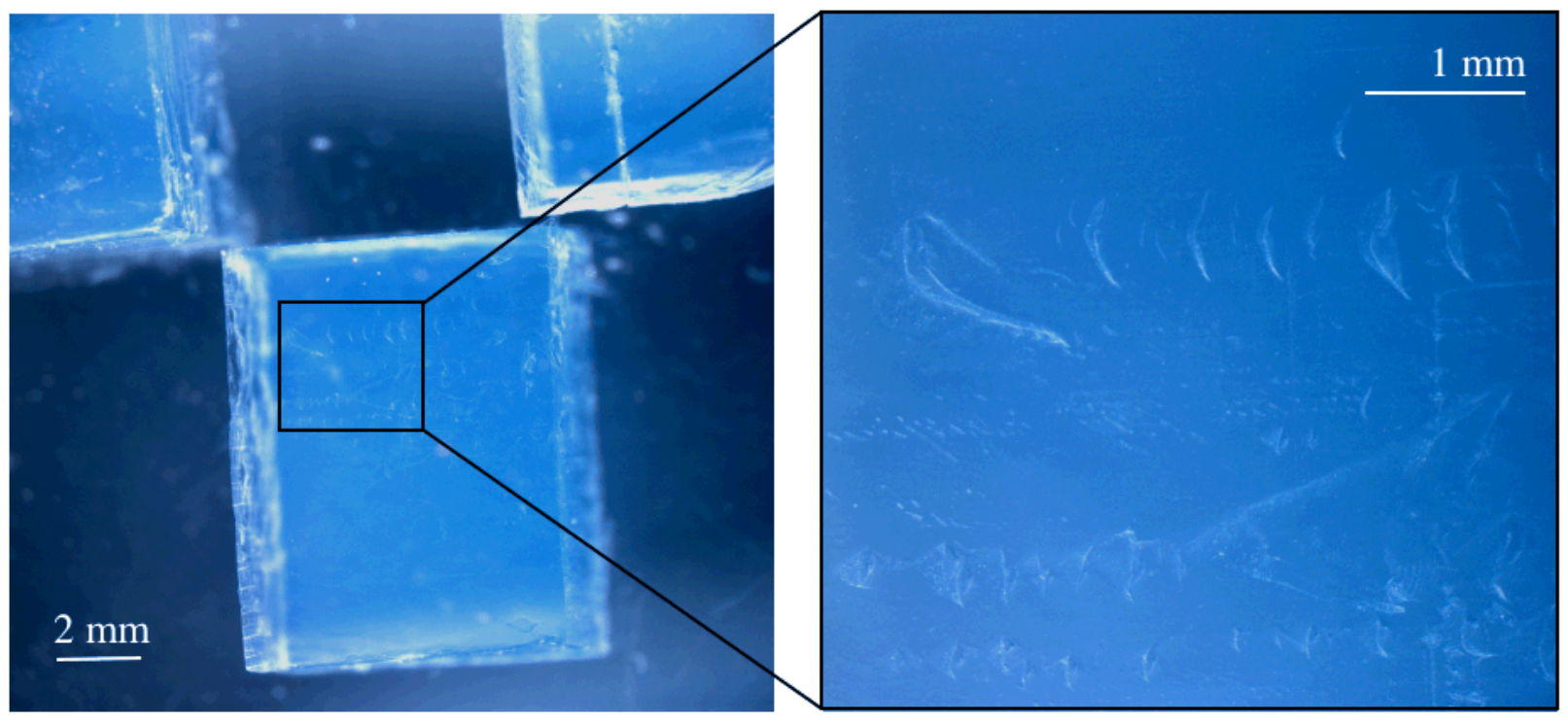

Figure 2. Silica aerogel block approximately $7.5 \times 7.5 \times 9 \mathrm{~mm}$ cut from the side of a tile using the ultrasonic macroblade in Figure 1. The block has fallen forward so that the top cut surface is the original cut back face. The cut surfaces have high optical clarity even with some tearing visible (right). Tearing typically initiates when too large steps are taken in depth during cutting. 


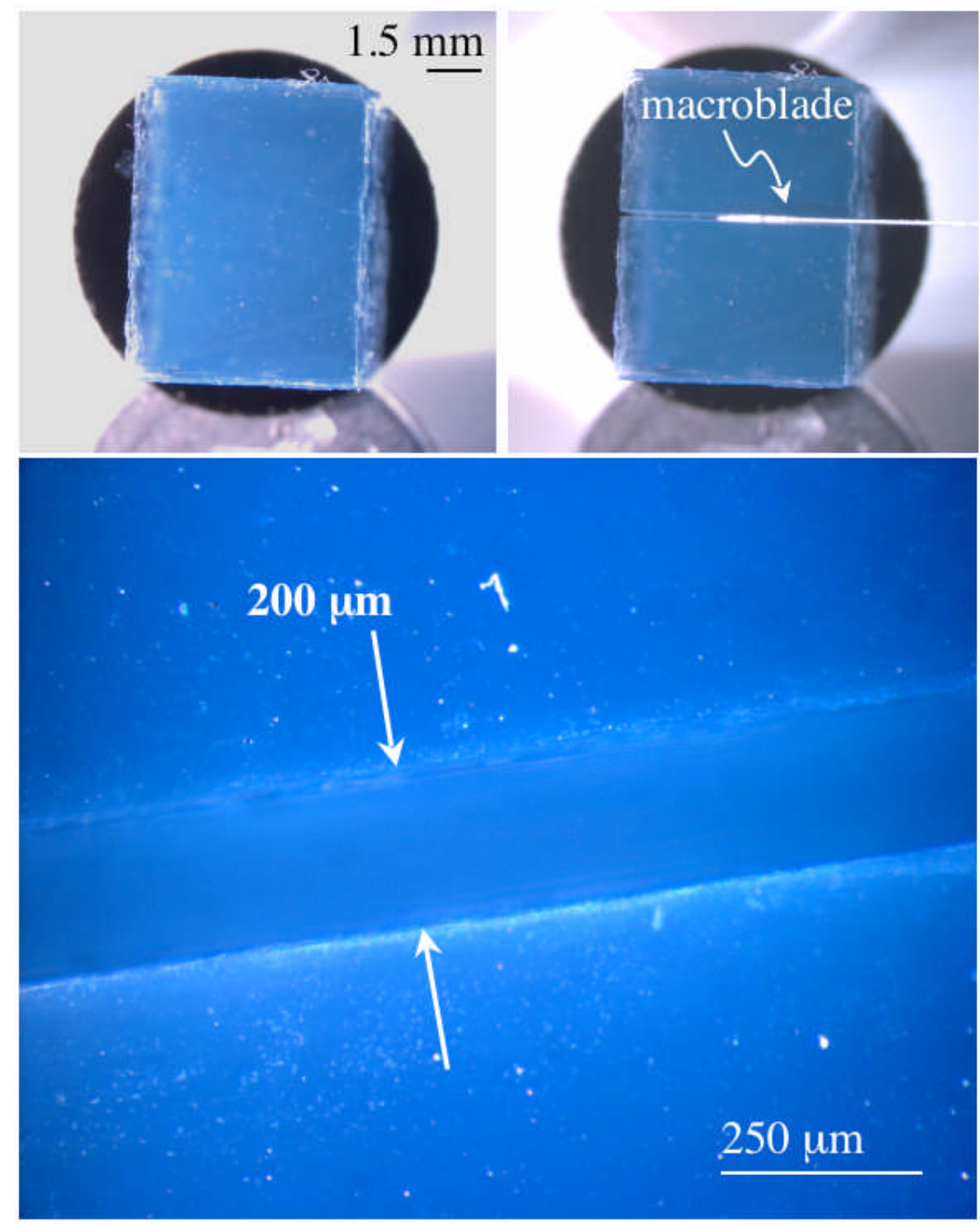

Figure 3. Large-scale cuts for embedding a microthermocouple in silica aerogel for synchrotron x-ray heating tests. Top left: The block cut from an edge of an aerogel tile mounted on a carbon adhesive pad. Top right: Cutting a groove with the steel macroblade and $\mathrm{U} / \mathrm{S}$ frequency vibrations. Bottom right: Closeup of the cut which extends downwards below the focal plane of the optical image. Because the aerogel is no longer constrained in its larger starting tile, the gap is $\sim 200$ microns wide reflecting the blade width and vibration amplitude. 